\title{
Astrometry and New Methods made possible by the new generation of telescopes
}

\author{
Maria J. Rioja*; Richard Dodson \\ ICRAR, Australia / OAN, Spain \\ E-mail: maria.rioja@icrar.org, richard.dodsoneicrar.org \\ Taehyun Jung, Bong Won Sohn \\ KASI, South Korea \\ E-mail: thjung@kasi.re.kr, bwsohn@kasi.re.kr
}

\begin{abstract}
Astrometric studies with VLBI have undergone an astonishing increase in astrophysical impact as a result of microarcsecond accuracies being routinely achieved in a moderate frequency regime using Phase Referencing techniques. This is largely driven by the development of advanced tropospheric calibration methods. We review alternative calibration approaches suitable to enable high precision astrometry in different frequency regimes, beyond the application of conventional Phase Referencing. That is, to observations at millimeter and submillimeter wavelengths (hereafter mmVLBI), to low frequencies, and to Space VLBI. Simultaneous multi-frequency observations and multiple calibrators are proposed as an effective strategy to eliminate the dominant atmospheric propagation effects at the highest and lowest observing frequencies, respectively. We present a demonstration with Korean VLBI Network observations at $132 \mathrm{GHz}(\sim 2 \mathrm{~mm})$ and simulation studies at low frequencies. This is particularly timely in the age of new instruments like ALMA, SKA and, potentially, the new Chinese-led Space VLBI mission.
\end{abstract}

12th European VLBI Network Symposium and Users Meeting,

7-10 October 2014

Cagliari, Italy

*Speaker. 


\section{Introduction}

The ability to measure positions is at the base of many astronomical and astrophysical studies, and VLBI provides the highest accuracy astrometric measurements in astronomy. The unmodelled propagation effects on the wavefronts from radio sources as they travel through the atmosphere constitute the dominant source of errors in VLBI observations, and are traditionally a major obstacle in astrometric measurements. More specifically, the tropospheric effects dominate in observations at high frequencies (as $\left.\phi_{t r p} \propto v\right)$, while ionospheric $\left(\phi_{i o n} \propto \lambda\right)$ dominate at low frequencies (with $v \sim 5 \mathrm{GHz}$ the cross over point where both are equal). Its compensation is mandatory for the recovery of the astrometric signature in the very precise interferometric phase observable in the data analysis. We review here strategies to enable astrometry in different observing frequency regimes, and the new opportunities in the light of the new generation of radio telescopes.

\subsection{Microarcsecond astrometry today at $\mathrm{cm}$-bands $(\sim 8-43 \mathrm{GHz})$, with PR techniques}

Currently, state-of-the-art microarcsecond ( $\mu$ as) relative astrometric measurements for a wide variety of targets are being achieved routinely, using Phase Referencing (hereafter PR) techniques. PR relies on using interleaving observations of a nearby calibrator or reference source to compensate for residual errors in the observations of the target source. In the early days of PR techniques, the application was limited to a few targets that fullfilled the stringent constraints required for succesful analysis, and $\mu$ as accuracies were limited to an even smaller subgroup of targets with an inbeam calibrator source, a configuration that provides a superior compensation of errors. This is no longer the case. Several factors that have contributed to the advances are: a significant improvement in telescope sensitivity from increased bandwidths and receiver quality, well maintained networks of telescopes with good coordinates, and improved "a priori" correlator models. The main contribution, however, comes from the development of advanced tropospheric calibration techniques which resulted in a leap forward [10,5]. It made possible the achievement of outcomes previously reserved for the rare configuration of targets; that is, accuracies which previously required an inbeam calibrator source now could be obtained with a reference source as far as $\sim$ a degree away, in $\mathrm{cm}$-wavelength VLBI observations. The general improvement in astrometric accuracy has resulted in much wider applications, to many astrophysical fields, as shown in the review presented by Reid \& Honma [11] (hereafter RH14) and references therein. Fig. 1 shows a qualitative description of the increasing astrophysical impact of VLBI astrometric studies, as it plots the average number of references per year listed in RH14.

Nevertheless, at this moment in time, the $\mu$ as astrometric measurements are limited in general to observations carried out with a ground array in a moderate frequency range ( $\sim 8-43 \mathrm{GHz})$. The reasons for this are: 1) At higher frequencies, the short tropospheric coherence times impose an impractically fast telescope switching rate; additionally, the intrinsic source fluxes are lower and the probabilities to find a suitable calibrator much reduced. The only successful demonstration beyond $43 \mathrm{GHz}$ was with the VLBA at 86-GHz for a pair of sources only 14' apart [8]. 2) At lower frequencies, the spatial structure of the dominant ionospheric contribution propagates into significant astrometric errors when using observations along different lines of sight, for the calibrator and target sources, in the PR analysis; Accurate, but not $\mu$ as level astrometry, has been achieved at 
frequencies down to $\sim 1.4 \mathrm{GHz}$ with in-beam PR (see references in RH14); 3) The application of PR techniques is even more restricted with Space-VLBI, where satellite operations are difficult and orbit determination errors, which are much larger than for the position of ground telescopes, can be the dominant contribution. Consequently there are only a few cases of limited in-beam astrometry with the Japanese satellite HALCA [7, 4].

\subsection{Microarcsecond astrometry with mm-VLBI, using SFPR technique.}

The fast tropospheric fluctuations and scarcity of suitable calibrator sources have prevented the application of PR techniques to $v>43 \mathrm{GHz}$, except for the single case at $86 \mathrm{GHz}$ previously mentioned. One alternative approach is to compensate for the dominant tropospheric residual errors by using dual frequency observations of the same target source. The tropospheric propagation introduces identical delays at both frequencies (i.e. a residual phase proportional to the observing frequency), because of its non-dispersive nature. Hence, the low frequency can be used to compensate for tropospheric residuals in the high frequency observations. This strategy was first attempted in VLBI by Middelberg et al. [6]; while this approach, which we dubbed Frequency Phase Transfer (hereafter FPT), serves to increase the coherence time in the high frequency observations as a result of removal of the tropospheric fluctuations, astrometry is not achieved. The remaining non-negligible dispersive ionospheric and instrumental errors prevent the extraction of the astrometric information (i.e. the Fourier Transform of the FPTed phases does not converge into a map). Interleaving observations of a second source, with a duty cycle up to many minutes, and angular separation up to several degrees, serves to compensate for the dispersive residuals and leads to high precision astrometric measurement between the observing bands. We dubbed this 2-step calibration astrometric strategy Source Frequency Phase Referencing (hereafter SFPR). The basis of the SFPR method, along with a comprehensive astrometric error analysis and observational demonstration has been presented elsewhere [13, 15]. The first observations were carried out in 2007 using VLBA fast frequency switching observations between 43 and $86 \mathrm{GHz}$, with a duty cycle of $\sim 1$ minute between frequencies. The analysis required careful manual data editing and was laborious. Given that a faster duty cycle would be required for higher observing frequencies, which is impractical, $86 \mathrm{GHz}$ is the limit of application with fast frequency switching observations. On the other hand, the Korean VLBI Network $(\mathrm{KVN})$ is a new dedicated mm-VLBI array equipped with multichannel receivers, which enable simultaneous observations at 4 frequency bands $(22,44,88,132$ $\mathrm{GHz}$ ). Simultaneous frequency observations are ideal for SFPR astrometry. A detailed comparative study of KVN simultaneous versus VLBA fast frequency switching observations at 22/44 GHz is presented by Rioja etal. [15]. Among the advantages of simultaneous observations are: a superior compensation of tropospheric (and in general, all non-dispersive) residuals, and suitability for (sub)mm-wavelengths. Such advantages are particularly relevant for VLBI with ALMA, both for achieving increased coherence time and astrometric measurements.

A successful demonstration of a related calibration strategy using dual frequency observations has been carried out with the ALMA interferometer for various frequency pairs from $100 \mathrm{GHz}$ up to $650 \mathrm{GHz}$ [2]. In Section 2 we describe a SFPR demonstration at the highest KVN band, at $2 \mathrm{~mm}$ (132 GHz). 


\subsection{Microarcsecond astrometry at the lowest frequencies, with MultiView VLBI technique}

The next generation of telescopes, such as the SKA, offers the exciting possibility to form multiple independently phased-up beams from the observing stations, which allows for the observation of multiple targets simultaneously. This could be important for deep VLBI surveys of sources close together on the sky, but the major application would be to improve the compensation of the propagation medium effects by using multiple calibrators and enabling high precision astrometry. As part of the VLBI Science Survey on ASKAP we are working towards implementing MultiView (or Cluster-Cluster) methods, i.e. using multiple calibrators, with Phased Arrays Feeds (PAF) as installed on the ASKAP array.

MultiView VLBI has been reviewed elsewhere [12] (see also ATMCA [3]), and here we only summarise the method and benefits. With multiple observations along different lines of sight we can solve for a full 2D correction of the spatial atmospheric distortions around the VLBI target, which will provide significantly improved calibration, imaging and astrometric precision compared to that from a single calibrator. This is particularly important in the ionospheric dominated regime $(<5 \mathrm{GHz})$. The improvement arises from the fitting of a linear surface to the calibration residuals and interpolating this model to the target position, which allows one to use calibrator sources with separations beyond the traditional ionospheric patch size (which is defined by the phase difference between the calibrator and target being greater than 1 radian). This separation can be extended even further if one solves for the phase ambiguities. Our simulation studies $[9,1]$ show that by using MultiView approaches with three calibrators one can achieve an order of magnitude improvement in the astrometric accuracy of the target, compared to using a single calibrator at similar angular separations.

\subsection{Microarcsecond astrometry with Space VLBI using new methods}

The extremely long baselines involved in Space VLBI hold the potential of achieving the highest spatial resolution. Nevertheless, the requirements for astrometry and enhanced sensitivity that are achieved with conventional PR using ground arrays are difficult to meet with a spacecraft. One of the greatest challenges is in the orbit reconstruction at the $\mathrm{cm}$-level. When the antenna is itself in a highly eliptical orbit of many thousands of kilometres at apogee this is an extremely challenging proposition. For the VSOP-2 project some solutions were put forward that held the promise of achieving these restrictive requirements, but these were both expensive and heavy. SFPR and MultiView have the potential to reduce the orbit reconstruction constraints significantly. In the higher frequency regime, dual frequency observations along with FPT or SFPR analysis result in cancellation of telescope position errors, since they have a non-dispersive nature [14]. In the low frequency regime, multi-directional observations towards $(\geq 3)$ calibrators surrounding the target source allows one to compute and eliminate the phase slope across the field of view introduced by orbit errors [1]. Therefore, Space VLBI observations with SFPR and MultiView techniques hold the potential of high accuracy astrometry and increased sensitivity in a wide frequency regime.

\section{Observations and Results from SFPR astrometry at $132 \mathrm{GHz}(2 \mathrm{~mm})$}

In March 5th 2014 we carried out KVN simultaneous observations at 22/44/88/132 GHz using SFPR astrometric techniques. The observations consisted of $\sim 3$ minute long scans alternating 
between the sources in each of the two triangles shown in Figure 2, and between the triangles ca. every hour. We followed the 2-step SFPR calibration technique for the analysis of each pair of sources, and for each pair of frequencies; the progressive calibration is shown with plots of the visibility phases. Figure 3 shows the raw visibility phases output of the correlator, at 22, 44, 88 and $132 \mathrm{GHz}$. The colours within each plot refer to observations of different sources. Figure 4 shows the FPTed calibrated phases at 44, 88 and $132 \mathrm{GHz}$, using $22 \mathrm{GHz}$ as the reference frequency; the FPT calibration consists of applying the antenna solutions obtained from the self-calibration analysis of observations at $22 \mathrm{GHz}$ (i.e. the reference frequency) of the same source, to the raw phases at 44, 88 and $132 \mathrm{GHz}$ (i.e. target frequencies), after scaling with factors 2, 4, and 6, respectively, with no further self-calibration. Fig. 5 shows a similar analysis for 88 and $132 \mathrm{GHz}$, using $44 \mathrm{GHz}$ as the reference frequency and with scaling factors 2 and 3, respectively. The FPTed calibrated phases show a much higher degree of coherence, compared to the raw phases, which translates into a significant increase of the coherence time, as expected after the random tropospheric fluctuations have been eliminated. The estimated coherence time at $132 \mathrm{GHz}$ is $\sim 20$ minutes after FPT calibration; after SFPR calibration, there is practically no limit. We could integrate for 8-hours, the whole duration of the experiment, with a mere $20 \%$ loss of peak flux. Nevertheless, the trends in the FPTed phases are dominated by remaining dispersive residuals, such as instrumental and ionospheric errors, which prevent direct imaging to extract the astrometric information. Astrometry is made possible with a second step of calibration which involves the interleaving observations of a second source. This step eliminates unwanted remaining dispersive residuals, while keeping the astrometric signature. Figs. 6 and 7 show the FPTed and SFPRed calibrated phases for two pairs of sources, $6.3^{\circ}$ and $11^{\circ}$ away, respectively, at $132 \mathrm{GHz}$. Fig. 6 shows the FPTed phases (two leftmost plots) for $1803+784$ and $2007+777$ at $132 \mathrm{GHz}$; the next plot to the right corresponds to the SFPRed phases of $2007+777$ at $132 \mathrm{GHz}$, after using $1803+784$ as the reference source; the $2007+777$ SFPRed dataset is Fourier transformed, without any other self-calibration step, to produce a SFPRed map at $132 \mathrm{GHz}$ (rightmost plot). Fig. 7 shows a similar sequence for the pair of sources with the largest separation, $1803+784$ and $1842+681,11^{\circ}$ away, at $132 \mathrm{GHz}$. The SFPRed maps are the final product of the astrometric analysis. Both maps are of high quality, with flux recoveries in the range 80-90\%; moreover it should be noted that none of the sources had direct detections at 132 GHz.

The SFPRed maps convey the astrometric measurements. The offsets of the peak of brightness from the center of the SFPRed map corresponds to a "bona fide" astrometric measurement of the shift between the maps at different frequencies, which is fundamental for spectral studies. The offset corresponds to the combined contribution from the two sources. We are developing a strategy to disentangle the individual contributions by using multiple sources and including the direction of the jet structure of the sources. The signal-to-noise ratios in the SFPRed maps shown in Figs. 6 and 7 are $\sim 30$ and 50, respectively, which translate into random astrometric errors of $\sim 10-20 \mu$ as.

\section{Discussion}

Simultaneous multi-frequency observations are presented as an effective strategy to eliminate the dominant tropospheric propagation effects which hinder mm-VLBI. The Korean VLBI Network 


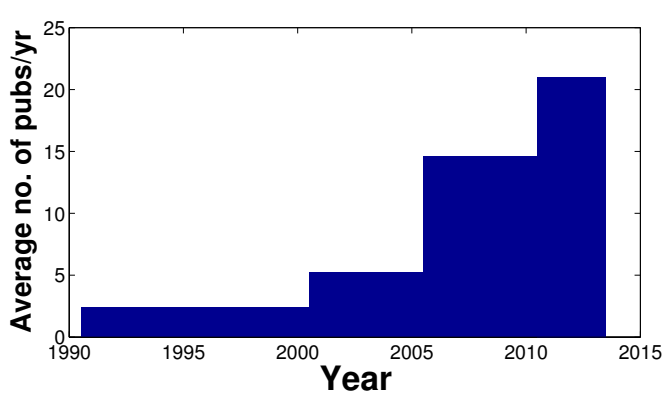

Figure 1: Histogram showing the increase in the average number of VLBI astrometry publications per year, compiled using the references listed in RH14. The increasing trend is correlated with the development of advanced tropospheric calibration techniques to complement PR analysis. This serves as a qualitative measurement of the astrophysical impact of routinely achieved $\mu$ as VLBI astrometry.
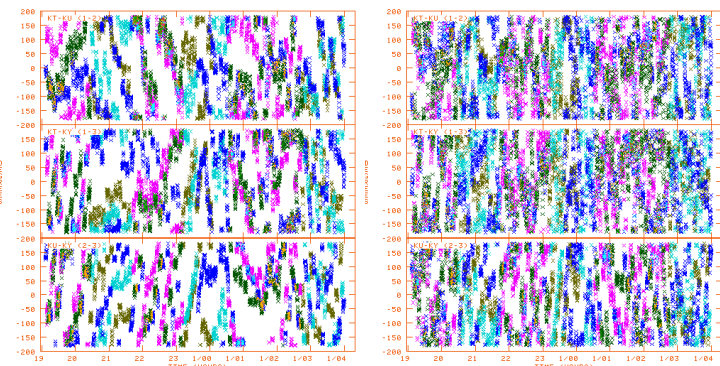

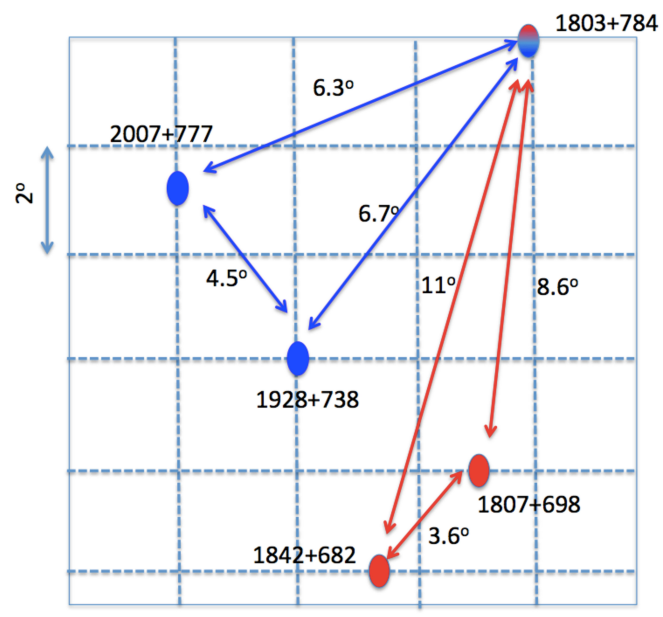

Figure 2: Sky Distribution of the two triangles of sources observed with the KVN simultaneously at 22/44/88/132 GHz, with $\sim 3$ min scans per source.
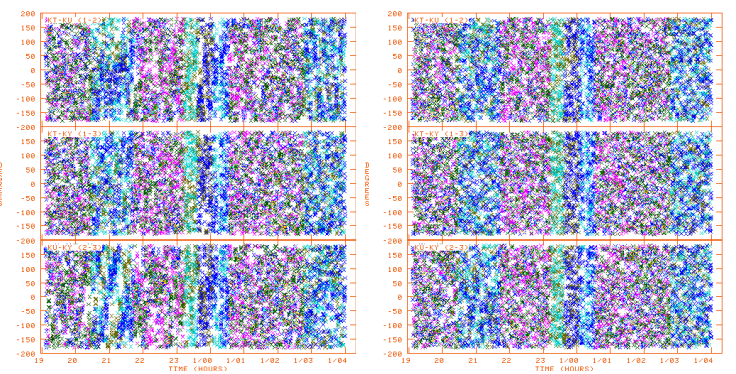

Figure 3: Raw residual visibility phases output of the correlator, from KVN observations at 22, 44, 88 and $132 \mathrm{GHz}$ described in this article. Different colors correspond to different sources.

was designed around this concept, and is equipped with multi-channel receiver systems which allow simultaneous observations from 22 up to $132 \mathrm{GHz}$. In this paper we have presented for the first-time a demonstration of high precision VLBI astrometric measurements at $2 \mathrm{~mm}$, using KVN observations and the SFPR technique, with source angular separations up to $11^{\circ}$. Dual frequency observations can play a key role to overcome the sensitivity limitation in mm-VLBI observations, by enlarging the coherence time. Therefore, the multi-frequency configuration opens a path for wider applications, to many sources, and for the foremost science topics with mm-VLBI.

The next generation of telescopes will provide new horizons for VLBI, particularly in the exploration of new frequency regimes. While the development of suitable advanced tropospheric calibration techniques have led to much wider applications for VLBI and improved astrophysical impact, their scope is limited to a moderate frequency regime. In this paper we have reviewed alternative VLBI calibration methods, namely SFPR and MultiView, that can operate at the frequency regimes of the new telescopes, such as ALMA and SKA, and in the presence of the large orbit errors of Space VLBI. Therefore, one can expect that they will contribute to expanding the impact 

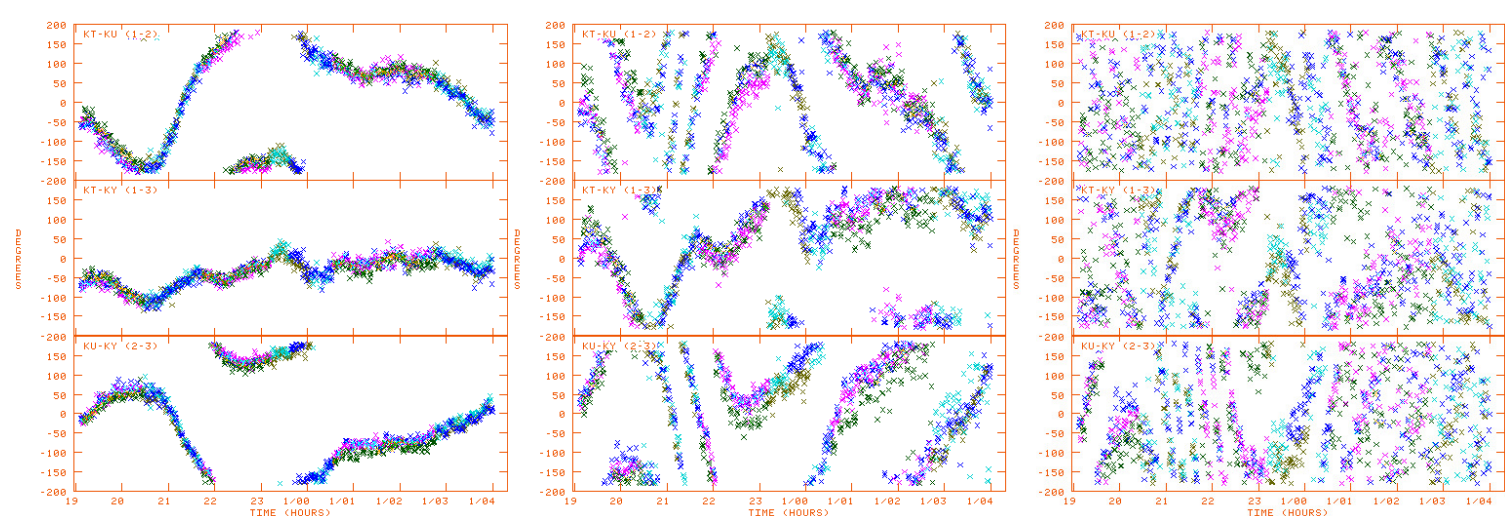

Figure 4: FPTed residual visibility phases at 44, 88 and $132 \mathrm{GHz}$, using $22 \mathrm{GHz}$ as the reference frequency, from KVN observations and FPT analysis. Different colors correspond to observations of different sources.
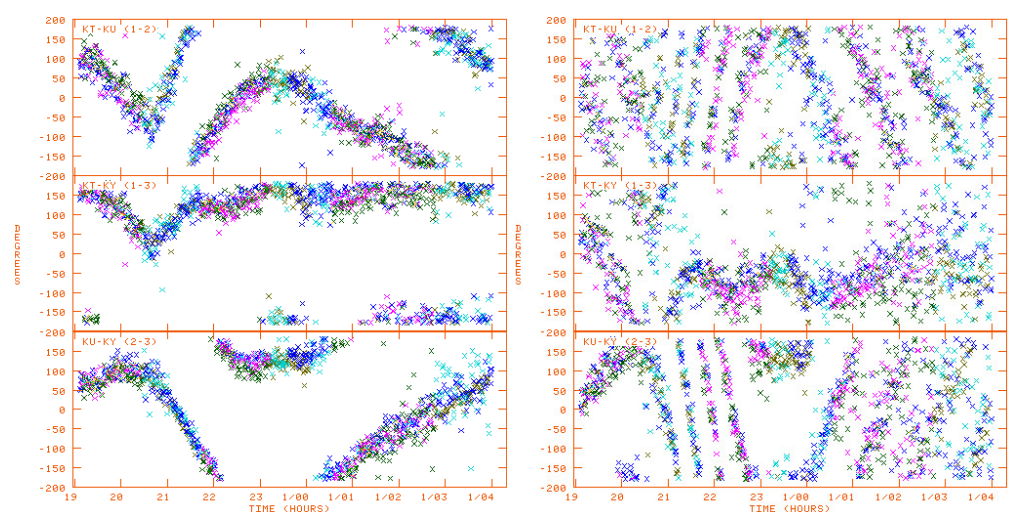

Figure 5: FPTed residual visibility phases at 88 and $132 \mathrm{GHz}$, using $44 \mathrm{GHz}$ as the reference frequency, from KVN observations and FPT analysis.

of VLBI in the era of the next generation telescopes.

SFPR KVN astrometric measurements are suitable for "bona fide" astrometric registration of images, both continuum and spectral line, at different frequencies, which are at the base of spectral studies. Nevertheless, some applications (i.e. AGN “core-shift" measurements), require long baselines to overcome dominant systematic errors, such as those arising from structure blending effects. The KVN has carried out joint observations with other telescopes equipped with the capability for simultaneous observations, such as VERA, with retro-fitted quasi-optic system at 22/44 $\mathrm{GHz}$, and ATCA in subarray mode at $44 / 88 \mathrm{GHz}$; very recently, Yebes-40m has been equipped with a simultaneous $22 / 44 \mathrm{GHz}$ system, and is also ready for joint observations which are expected shortly. We expect more telescopes will be available in the near future. There is a great potential for a network of telescopes with the capability for simultaneous multi-frequency observations at high frequencies, and with long baselines, to tackle the goals proposed for EHT and Space VLBI projects, at significantly lower cost. 

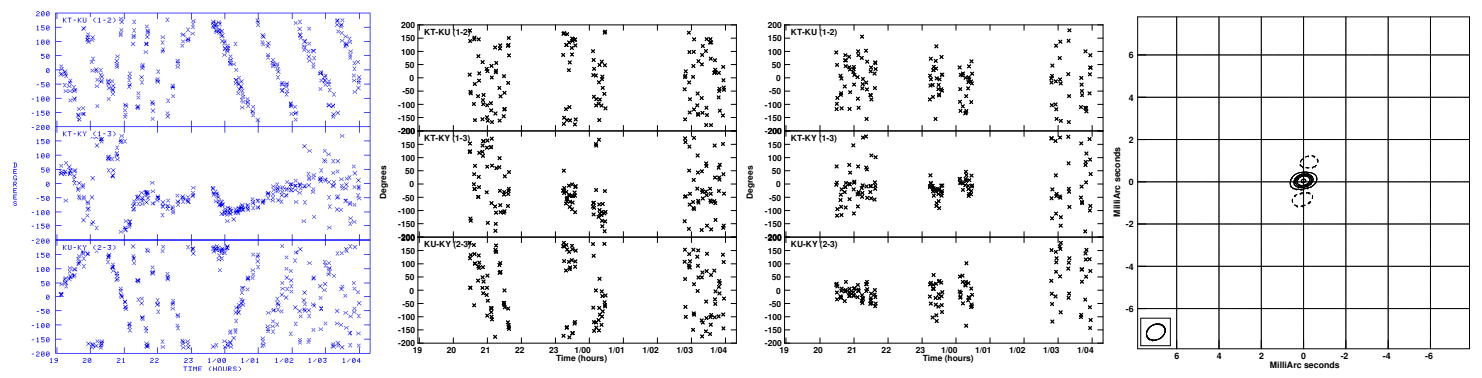

Figure 6: (First and Second plots) FPTed residual visibility phases at $132 \mathrm{GHz}$, for $1803+784$ and 2007+777, respectively, with $44 \mathrm{GHz}$ as reference frequency; (Third plot) SFPRed residual visibility phases at $132 \mathrm{GHz}$ for $2007+777$ using $1803+784$ as the reference source, $6.3^{\circ}$ away, and $44 \mathrm{GHz}$ as the reference frequency; (Fourth plot): SFPRed map of 2007+777 at $132 \mathrm{GHz}$, from KVN observations and SFPR analysis.
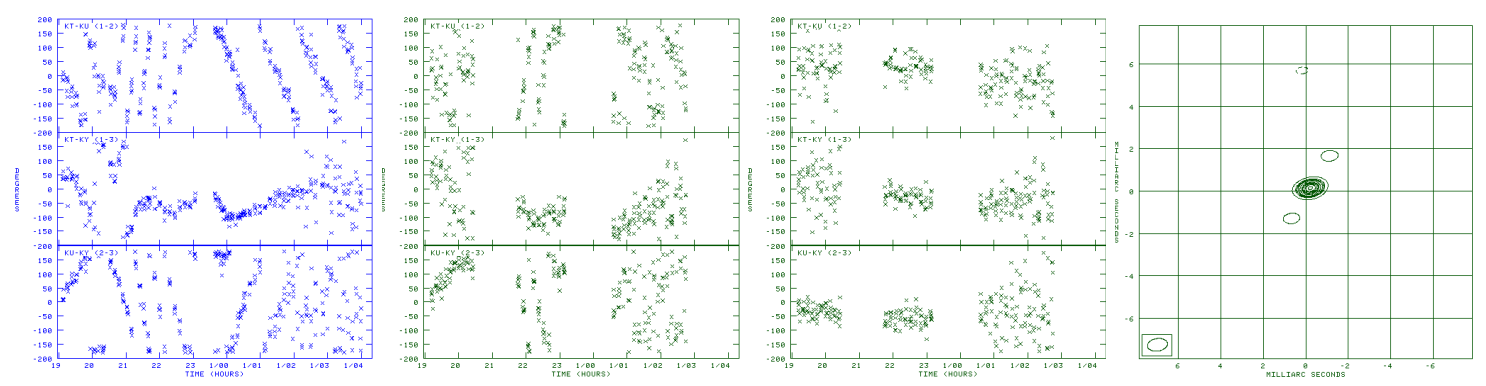

Figure 7: Results from SFPR astrometric analysis at $132 \mathrm{GHz}$ for the most widely separated pair of sources, $\sim 11^{\circ}$ away. Same as Figure 6, with 1803+784 (as reference source) and 1842+681 (as target source).

\section{References}

[1] R. Dodson, M. Rioja et al., 2013, AJ 145 (147)

[2] E. Fomalont, V. Impellizzeri, C. Wilson, 2014, Implementation of BandtoBand Phase Transfer, EOC Memo 13

[3] E. Fomalont, 2005, Phase Ref. Using More Than 1 Calibrator, Fut. Dir. in High Res. Astronomy, 340, 460

[4] J. C. Guirado, et al., 2001, $A \& A 371$ (766)

[5] M., Honma, Y., Tamura, \& M. J., Reid, 2008, PASJ 60 (951)

[6] E. Middelberg, A. Roy, R. C. Walker \& H. Falcke, 2005, AA 433 (897)

[7] R. W. Porcas, M. J. Rioja, J. Machalski, \& H. Hirabayashi, 2000, Astrophysical Phenomena Revealed by Space VLBI, ed. H. Hirabayashi, P. G. Edwards, \& D. W. Murphy (245)

[8] R. W. Porcas, \& M. J. Rioja, 2002, Proc. 6th EVN Symp. (65)

[9] S. Jiménez-Monferrer, M. J. Rioja, R. Dodson et al., 2010, Proc. 10th EVN Symposium

[10] M. J., Reid, K. M. Menten, A., Brunthaler, et al. 2009, ApJ 693 (397)

[11] M. J. Reid \& M. Honma, 2014, Microarsecond Radio Astrometry, Annu. Rev. Astro. Astrophysics 52 (339)

[12] M. J. Rioja, R. Dodson, et al., 2009, Proc. 8th International e-VLBI Workshop

[13] M. J. Rioja \& R. Dodson, 2011, High Precision mm-VLBI using a new method for calibration, AJ 141 (114)

[14] M. J. Rioja, R. Dodson, J. Malarecki, Y. Asaki, 2011, AJ 142 (157)

[15] M. J. Rioja, R. Dodson, + KVN Team, 2014, AJ 148 (84) 